\title{
Animal mutants and medical genetics: hereditary disorders of mouse erythropoiesis as experimental tools
}

\author{
R. J. COLE \\ Ph.D. \\ School of Biological Sciences, University of Sussex
}

\section{Introduction}

Homeostasis in the mammalian erythroid system at all stages of development depends on the rate of formation and survival of new erythrocytes following the efficient functioning of balancing regulators such as the rate of production of, and the response to, erythropoietin. A variety of inherited anaemias, each representing a separate disruption of erythroid regulation, may be used as experimental tools in the analysis of these mechanisms. Twelve single-geneinduced anaemias have been described in mice, a number of which have close similarities to human anaemias (reviews, Russell \& Bernstein, 1966, Pinkerton \& Bannerman, 1968; Bernstein, 1969; Russell, 1970). Analysis of gene action in mammals presumes that each of the alleles at a given locus affects the same original process, usually synthesis of a specific protein, and a particular gene may affect the metabolism of only one cell-type in a differentiated organism. Genes at different loci affect different processes so that each single-gene-induced anaemia should show abnormality of a different specific process.

Recent advances in studies of the kinetic properties of normal erythroid systems provide the background against which the effects of mutant gene action can be assessed.

\section{Kinetic properties of the normal erythroid system}

The kinetic properties of the erythroid system can be described in Fig. 1. The parameters defining each compartment are $N$, the number of cells; $f$ in, the inflow of cells; $F$ out, the outflow of cells; $\alpha$, fractional birthrate; $\beta$, fractional death rate; $m$, number of divisions; $T$, transit time; and $t_{\mathrm{c}}$, cell cycle time Fig. 2 . The nature of compensatory events in such a system can be investigated in animals which have reached a new steady state in response to stress applied at a

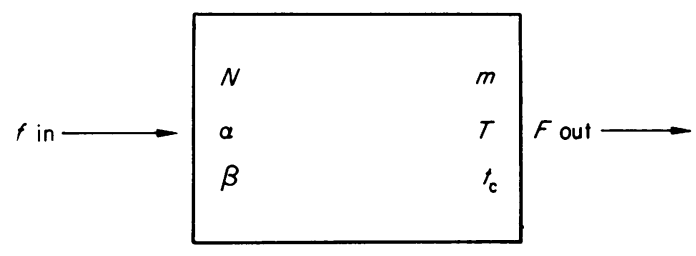

Fig. 2 .

constant rate. Cell population kinetic studies of the recognizable part of the erythroid system in chronically irradiated and chronically anaemic rats show that compensation can take place in different parts of the erythron (Tarbutt, 1969).

Reduced inflow $\left(f /\left.\right|_{2}-f / 4\right)$ into the recognizable erythroid precursor compartments caused by irradiation is compensated for by extra divisions during erythroblast maturation, at the expense of increased transit time. Enhanced outflow $(5 F)$ of erythrocytes in chronic anaemia is met by increased inflow $(4 f)$ of cells into the proerythroblast compartment, and reduction of cell cycle times during maturation.

\section{Prenatal erythropoiesis in mice}

Recent studies have established that prenatal erythropoiesis is regulated by feed-back controls similar to those operating in the adult and may be even more vulnerable to the effect of mutant genes. A small number of haemopoietic stem cells are present in the mouse liver on the tenth day of gestation, either arising endogenously or migrating in from another site. These cells can be recognized by their ability to form colonies in the spleens of irradiated recipient mice (Niewisch et al., 1970). During the hepatic phase of erythropoiesis, the number of hepatogenic red cells in the circulation increases in two distinct exponential phases with doubling times of 8 and $48 \mathrm{hr}$ respectively (Paul, Conkie \& Freshney, 1968) with a transition about the

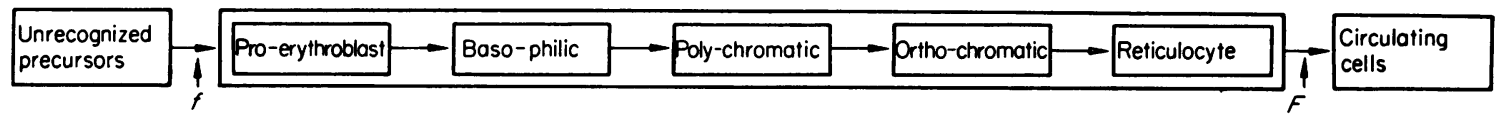

FIG. 1. 
fifteenth day of development. During the period of rapid increase, erythroblasts taken from foetal livers can be stimulated to increased haem synthesis in vitro by erythropoietin, but during the later period, cultures are refractory to further stimulation (Cole, Hunter \& Paul, 1968). The sudden transition from a fast-growing to a slow-growing erythrocyte population reflects changes in the precursor population in the liver. In the first phase, the cell cycle time of early erythroid precursors is about $5 \frac{1}{2} \mathrm{hr}$, and of late precursors about $8 \mathrm{hr}$. In the later phase of hepatic erythropoiesis, both early and late precursors have a $5 \frac{1}{2}$-hr cell cycle time (Tarbutt \& Cole, 1970). The transition in the rate at which erythrocytes appear in the foetal circulation is due to a decrease in the absolute number of erythroid cells in the liver, and the proportion of cells capable of proliferation.

The kinetic behaviour of normal foetal liver erythroid cell populations therefore appears to be determined by their reaction to two stress situations acting concurrently. The first, analogous to that in the continuously irradiated adult rat, results from the limited number of 'stem cells' available to feed into the differentiating cell population. The second is analogous to that in the chronically phenylhydrazineanaemic adult and results from the increasing tissue mass of the rapidly growing foetus, which has to be supplied by the foetal erythron.

\section{Genetic effects on the erythroid system}

Studies of erythropoiesis at the tissue, cellular, and molecular levels have disclosed a number of possible sites of action of anaemia-producing mutant genes. Initiation of erythropoiesis could be restricted by genes which reduce the number of totipotent stem cells or of erythropoietin-sensitive cells, or reduce their responsiveness to stimuli to growth or differentiation. Maturation of erythroid cells can be impaired by intrinsic defects such as reduction in the supply of their substrates due to deficient absorption or transport, or restriction on cell multiplication. Genetically determined extrinsic factors may also affect survival and differentiation of erythroid cells, e.g. by affecting matrix cells or by reduction in metabolic supply. Several genetically determined properties can potentially reduce the lifespan of circulating peripheral cells, e.g. membrane fragility, unstable haemoglobins and enzyme deficiencies.

Some anaemia-producing abnormalities may therefore be restricted to a single stage of development and a single tissue. Since erythroid homeostasis is maintained by feedback controls, compensation may be more likely when mutations induce peripheral cell defects, such as reduced red cell lifespan, than when initiation of erythropoiesis or erythroid cell maturation are affected.

Mouse-anaemia mutants, in common with other animal mutants of potential interest to clinicians as 'model' systems fall into two main classes. In the first class, the animal mutant has such a close biochemical similarity to the human condition, coupled with the ease of obtaining material with a constant genetic background, that it can be used as a 'prototype' for experimental studies. The second class consists of mutants in which the genetic lesion may be used as a tool to disturb a closely regulated homeostatic system. Examination of the response to such perturbation assists detailed analysis of the control system which, in Man, may be subject to genetic damage at a variety of points.

In contrast to the situation in Man, where at present the clinically most important forms of inherited anaemias are associated with abnormal haemoglobins, the most studied inherited anaemias in mice involve abnormalities of haemopoietic cell proliferation.

The most important mutants will be briefly described and the main theme will be illustrated by a more detailed discussion of the flexed $(f / f)$ mutant.

Tail-short anaemia ( $T s)$, Hertwig's anaemia (an), diminutive $(\mathrm{dm})$, and dominant spotting ( $W$ series)

TABLE 1. Inherited defects in the mouse erythron

\begin{tabular}{clc}
\hline Developmental Stage & \multicolumn{1}{c}{ Nature } & Gene \\
\hline Prenatal & Stem-cell deficiency & $W / T s / d m$ \\
& Cell maturation + iron utilization & $f$ \\
& Extra-erythroid environment & $S l$ \\
Neonatal & Stem-cell deficiency & $W / d m$ \\
& Extra-erythroid environment & $S l$ \\
& Iron utilization & $\mathrm{f} / \mathrm{mk}$ \\
& Globin synthesis & $\mathrm{mk}$ \\
& Erythroid membrane defects & $\mathrm{ha} / \mathrm{ja} / \mathrm{nb}$ \\
& Blood loss & $\mathrm{lx}$ \\
& Stem-cell deficiency & $\mathrm{W} / \mathrm{dm}$ \\
& Adult & $\mathrm{Sl}$ \\
& Extra-erythroid environment & $\mathrm{f} / \mathrm{mk}$ \\
& Iron utilization & $j a / \mathrm{ha} / \mathrm{nb}$ \\
\hline
\end{tabular}


are all characterized by deficiencies in stem-cells during prenatal life. $T s$ is transitory, affecting only embryonic mice, appearing as a reduced number of blood islands in the yolk sac with secondary effects on the skeletal system. Hertwig's anaemia, (an) appears first on day 12, and is severe so that not all mice survive to weaning; it appears to be caused by delayed multiplication of haematopoietic colonyforming units. Diminutive homozygotes $d m / d m$, are born with one-third normal red cell number. The cells are macrocytic, and only half the normal number of colony-forming units are present in the bone marrow. Presence of two $W$ alleles causes a continuing severe macrocytic normochromic anaemia, apparent by the twelfth day of gestation and sometimes lethal. There is a deficiency in the capacity of stem cells to replicate and differentiate, the anaemics are markedly unresponsive to erythropoietin, and there is a 500-fold deficiency in erythroid colony-forming units in the marrow. $W$ anaemics can be 'cured' by transplantation of normal haemopoietic tissues, showing that the defect is intrinsic to the erythroid stem-cell.

The peripheral blood picture of viable combinations of steel genes, e.g. $\left(S l / S l^{d}\right)$ is identical to that of the $W$ series and they are phenotypically indistinguishable from $W$ anaemics; being black-eyed, white, sterile, macrocytic, normochromic anaemics, both very unresponsive to erythropoietin. Although the steel marrow is hypoplastic, the proportion of marrow colony-forming units is not reduced and steel erythroid tissue is capable of normal proliferation and differentiation when transplanted to a nonsteel environment.

Sex-linked anaemia (sla) is caused by a disorder of iron supply and transport, which gives rise to a 'target cell' hypochromic microcytic anaemia of embryo, newborn and adult, together with a deficiency of erythropoietic cells in the bone marrow. Spherocytosis (sph), jaundiced ( $j a)$, haemolytic anaemia $(h a)$ and normoblastic anaemia $(n b)$ are all caused by changes in the properties of erythroid cell membranes leading to haemolytic anaemia. In the case of $n b / n b$, an early erythroid cell type appears to be affected. In microcytic anaemia $(m k)$ iron utilization appears to be restricted and there is also haemolysis.

\section{The anaemia of flexed tailed $(f / f)$ mice}

Prenatal and neonatal homozygous flexed mice have a severe hypochromic microcytic anaemia, characterized by a high frequency of siderocytes, and their growth is retarded (Gruneberg, 1942). Blood values of adult $f \mid f$ mice are normal but their response to artificially-induced anaemia is delayed (Coleman, Russell \& Levin, 1969). Several similarities exist between the effects of the flexed mutation and the hereditary sideroblastic anaemia of man (Losowsky \& Hall, 1965) and some features of the di Guglielmo syndrome. Recent studies (Bateman \& Cole, 1972; Bateman et al., 1972; Cole, Regan \& Tarbutt, 1972) have shown that the severe anaemia of newborn $f / f$ mice, with one-third the normal haemoglobin complement per unit of body weight, results from complex interactions between effects on cell proliferation, and reduced haemoglobin synthesis in reticulocytes.

The earliest difference detectable between prenatal flexed and normal mice is a deficiency of colonyforming cells in the liver of 12-day foetuses (Bateman \& Cole, 1972). A deficiency probably exists earlier than this, but is below the resolution of the assay method. This deficiency lasts until day 16 , when numbers of c.f.u. become normal. The differentiative capacity of early foetal liver c.f.u. measured by ${ }^{59} \mathrm{Fe}$ uptake into blood cells 7 days after transplantation into irradiated hosts is also less than that of normal stem cells. The behaviour of $f / f$ stem cells in the early foetal liver is therefore similar to that in spleen colonies (Fowler et al., 1967) and some features of the $f / f$ lesion can be explained by an initially slow growth rate of stem cells so that $f / f$ animals are unable to respond efficiently to erythropoietic stress.

The deficiency in colony-forming cells is followed by retarded development of the erythroid cell popu- 은 lation in the $f / f$ foetal liver (Bateman et al., 1972). From day 12 to $17, f \mid f$ foetal livers have fewer cells than normal, and in addition contain a smaller proportion of erythroblasts, although erythropoiesis persists longer in the livers of $f / f$ than normal foetuses. Maturation of the foetal liver as an erythroid organ is also delayed in $f / f$. In normal foetuses late erythroblasts exceed early erythroblasts before day 12 , but in $f / f$ this does not occur until the thirteenth day. The deficiency in erythroblasts is such that on day 12 haem synthesis in $f / f$ livers is only $6 \%$ that in normals, although on a per cell basis haem synthesis appears to be normal in the nucleated cell population.

Although the number of erythroblasts is reduced in early $f / f$ livers, changes in the cell populations indicate that they may be proliferating at a higher rate than normal, suggesting an enhanced response to deficiency in the input of precursor cells, as seen in the continuously irradiated rat. Detailed cell kinetic studies of $f \mid f$ and normal foetuses (Tarbutt \& Cole, unpublished) show that the lengthening of the cell cycle in late erythroblasts found in the early stages of hepatic erythropoiesis, does not occur in $f / f$ foetuses. Evidence of enhanced cell proliferation in $f / f$ erythroblasts is also seen when the cells are explanted. More erythroid cells are produced in $f / f$ cultures in response to erythropoietin than in control 
cultures of normal cells, and the rate of DNA synthesis is higher. Studies of DNA and RNA and haem synthesis in $f \mid f$ foetal liver cells stimulated in vitro with erythropoietin show that the responses of $f / f$ erythroblasts to this hormone are unimpaired and may even be increased (Bateman et al., 1972).

The properties of $f / f$ reticulocytes are in marked contrast to the normality of metabolic patterns in nucleated erythroblasts. Lesions in haemoglobin synthesis in reticulocytes, during which stage two thirds of the cellular complement of haemoblobin is normally synthesized, is a major cause of the severe anaemia of neonatal $f / f$ mice (Cole et al., 1972). Iron uptake into $f / f$ late foetal reticulocytes is apparently normal but in comparison with normal reticulocytes, they utilize only half as much of this iron for haem synthesis. Estimation of the intracellular iron pool in late foetal reticulocytes by isotope dilution methods shows that in spite of the large deposits of iron present in the siderotic granules of $f / f$ reticulocytes, the amount which can be made available for haem synthesis is considerably less than in normal reticulocytes. On day 18 of gestation, $f / f$ reticulocytes contain $0.2 \mu \mathrm{g}$ of available iron $/ 10^{7}$ cells while normal reticulocytes contain $0.5 \mu \mathrm{g} / 10^{7}$ cells. These results point to severe disruption of haem synthesis in $f / f$ prenatal reticulocytes, but addition of haemin to $f / f$ reticulocytes in vitro does not lead to increased globin or haemoglobin synthesis and it appears that protein synthesis proceeds at a near normal rate to produce an excess of free globin chains. A similar situation has also recently been described in reticulocytes of human sideroblastic anaemia (White, Brain \& Ali, 1971). Abnormalities of iron metabolism may affect haem synthesis terminally by reducing the activity of iron chelatase (haem synthetase) but iron has also shown to be necessary for the activity of $\delta$-amino-laevulinic acid synthetase, which may be the critically controlled rate-limiting step in haem synthesis in all tissues. There is as yet no satisfactory explanation of a causal link between the failure of haemoglobin synthesis in the terminally differentiated prenatal reticulocyte and the retarded colonization of the foetal liver by the earliest precursor cells, followed by compensatory enhanced proliferation of erythroblasts. Although the response of adult $f / f$ mice to artificially-induced anaemia is delayed, even severely stressed $f / f$ adults produce circulating reticulocytes with normal synthetic patterns indicating that the synthetic lesion may result from conditions of cellular proliferation specific to the hepatic phase of erythropoiesis.

\section{Conclusions}

While many of the twelve well-characterized mouse mutations affecting the erythron are phenotypically similar, the primary action of each gene is unique, affecting a single point in the homeostatic regulation of blood-cell formation and destruction. The existence of this series of mutants therefore provides an opportunity to identify and analyse single steps in regulation of erythropoiesis. As models of human diseases some hereditary mouse anaemias already provide useful analogies, while others require further investigation before their potential in this respect can be exploited. In some cases the human counterpart to clearly defined inherited anaemia syndromes of the mouse remains to be described. Studies with these mutants underlines the susceptibility of the prenatal erythropoietic system to disturbance as a result of mutant gene action, and similar sensitivity might well exist in the human foetus. Widespread skeletal and other abnormalities, persisting throughout life, can result from genetically determined but transient disturbance of erythropoiesis at critical stages of embryogenesis, and some congenital abnormalities in Man might result from similar interactions.

\section{Acknowledgments}

Original work reported here was done in collaboration with Dr R. G. Tarbutt and Dr A. E. Bateman, with the support of the Medical Research Council and Science Research Council.

\section{References}

Bateman, A.E. \& Cole, R.J. (1972) Colony forming cells in the livers of prenatal flexed (f/f) anaemic mice. Cell and Tissue Kinetics (in press).

Bateman, A.E., Cole, R.J., Regan, T. \& Tarbutr, R.R. (1972) The role of erythropoietin in prenatal erythropoiesis of flexed tailed (f/f) mice. British Journal of Haematology (in press).

BERNSTEIN, S.E. (1969) Hereditary disorders of the rodent erythron. In: Genetics in Laboratory Animal Medicine. National Academy of Science (publication 1679), Washington, D.C.

Cole, R.J., Hunter, J. \& Paul, J. (1968) Hormonal regulation of foetal haemoglobin synthesis. British Journal of Haematology, 14, 477.

Cole, R.J., REgaN, T. \& TARBUTt, R.G. (1972) Haemoglobin synthesis in reticulocytes of prenatal $\mathbf{f} / \mathbf{f}$ mice. British Journal of Haematology (in press).

Coleman, D.L., Russell, E.S. \& Levin, E.Y. (1969) Enzymatic studies of the hemopoietic defect in flexed mice. Genetics, 61, 631.

Fowler, J.H., Till, J.E., McCulloch, E.A. \& Siminovitch, L. (1967) The cellular basis for the defect in haemopoiesis in flexed tailed mice. British Journal of Haematology, 13, 250.

GRUNeberG, H. (1942) The anaemia of flexed tailed mice. Journal of Genetics, 44, 246.

Losowsky, M. \& HALL, R. (1965) Hereditary sideroblastic anaemia. British Journal of Haematology, 11, 70.

Niewisch, H., Hajdick, I., Sultanian, I., Vogel, H. \& Matiol, G. (1970) Hemopoietic stem cell distribution in tissues of fetal and newborn mice. Journal of Cell Physio$\log y, 76,107$.

Paul, J., ConkIE, D. \& Freshney, R.I. (1969) Erythropoietic cell population changes during the hepatic phase of erythropoiesis in the foetal mouse. Cell and Tissue Kinetics, $2,283$. 
Pinkerton, P.H. \& Bannerman, R.M. (1968) The hereditary anaemias of mice. Hematologic Reviews, 1, 119.

RUSSELL, E.S. (1970) Abnormalities of erythropoiesis associated with mutant genes in mice. In: Regulation of Erythropoiesis (Ed. by A. S. Gordon) Vol. 1. Appleton-CenturyCrofts, New York.

Russell, E.S. \& Bernstein, S.E. (1966). Blood and blood formation. In: Biology of the Laboratory Mouse (Ed. by E. L. Green), 2nd edn. McGraw Hill, New York.
TARBUTt, R.G. (1969) Cell population kinetics of the erythroid system in the rat. British Journal of Haematology, $16,9$.

TARBUTt, R.G. \& Cole, R.J. (1970) Cell population kinetics in the livers of foetal mice. Journal of Embryology and Experimental Morphology, 24, 249.

White, J.M., Brain, M.C. \& Ali, M.A.M. (1971) Globin synthesis in sideroblastic anaemia. British Journal of Haematology. 20263. 